Article

\title{
POSS-Derived Synthesis and Full Life Structural Analysis of Si@C as Anode Material in Lithium Ion Battery
}

\author{
Ziyu Bai ${ }^{1}$, Wenmao Tu ${ }^{1, *}$, Junke Zhu ${ }^{1}$, Junsheng $\mathrm{Li}^{2, *}{ }^{\mathbb{C}}$, Zhao Deng ${ }^{1}$, Danpeng $\mathrm{Li}^{1}$ \\ and Haolin Tang 1,*(D) \\ 1 State Key Laboratory of Advanced Technology for Materials Synthesis and Processing, \\ Wuhan University of Technology, Wuhan 430070, China; 18971142560@163.com (Z.B.); \\ bebetterzjk@163.com (J.Z.); dengzhao@whut.edu.cn (Z.D.); 18435138790@163.com (D.L.) \\ 2 School of Chemistry, Chemical Engineering and Life Sciences, Wuhan University of Technology, \\ Wuhan 430070, China \\ * Correspondence: tuwm@whut.edu.cn (W.T.); li_j@whut.edu.cn (J.L.); thln@whut.edu.cn (H.T.)
}

Received: 15 March 2019; Accepted: 26 March 2019; Published: 29 March 2019

\begin{abstract}
Polyhedral oligomeric silsesquioxane (POSS)-derived Si@C anode material is prepared by the copolymerization of octavinyl-polyhedral oligomeric silsesquioxane (octavinyl-POSS) and styrene. Octavinyl-polyhedral oligomeric silsesquioxane has an inorganic core $\left(-\mathrm{Si}_{8} \mathrm{O}_{12}\right)$ and an organic vinyl shell. Carbonization of the core-shell structured organic-inorganic hybrid precursor results in the formation of carbon protected Si-based anode material applicable for lithium ion battery. The initial discharge capacity of the battery based on the as-obtained Si@C material Si reaches $1500 \mathrm{mAh} \mathrm{g}^{-1}$. After 550 charge-discharge cycles, a high capacity of $1430 \mathrm{mAh} \mathrm{g}^{-1}$ was maintained. A combined $\mathrm{XRD}, \mathrm{XPS}$ and TEM analysis was performed to investigate the variation of the discharge performance during the cycling experiments. The results show that the decrease in discharge capacity in the first few cycles is related to the formation of solid electrolyte interphase (SEI). The subsequent rise in the capacity can be ascribed to the gradual morphology evolution of the anode material and the loss of capacity after long-term cycles is due to the structural pulverization of silicon within the electrode. Our results not only show the high potential of the novel electrode material but also provide insight into the dynamic features of the material during battery cycling, which is useful for the future design of high-performance electrode material.
\end{abstract}

Keywords: Octavinyl-POSS; organic-inorganic crosslinking; Si@C anode; lithium ion battery; mechanism analysis

\section{Introduction}

Owing to the aggravation of energy crisis, the demand for new energy conversion and storage devices is growing continuously in recent years. As a promising chemical power supply for electronic device and electric vehicles [1,2], the lithium ion battery has found enormous applications in a variety of applications due to its advantages of high energy storage density and high open circuit voltage $[3,4]$. At present, graphite carbon is used as negative electrode in the commercialized lithium ion battery system. The theoretical capacity of graphite is only $372 \mathrm{mAh} \mathrm{g}^{-1}$ and replacing graphite in the anode with a robust material of high capacity may lead to a battery with higher energy density [5-9]. The theoretical specific capacity of silicon is up to $4200 \mathrm{mAh} \mathrm{g}^{-1}$, one order of magnitude higher than that of graphite anode material. Furthermore, the insertion/deinsertion potential of lithium ion for silicon is moderate [10,11]. Due to these advantages, a silicon-based material is an ideal choice for the next generation of lithium ion battery anode. However, during the alloying reaction of silicon 
with lithium, silicon material will suffer severe volume expansion, which can easily lead to rapid pulverization and detachment of the active material from current collector, which causes the rapid decline of the battery performance [12]. Meanwhile, the silicon material cannot form stable solid electrolyte interface (SEI). The newly exposed silicon surface due to the pulverization will continuously form a new SEI film, which leads to the decrease of charge and discharge efficiency and the acceleration of capacity attenuation [13].

In order to tackle the above-mentioned problems, the following strategies have been made in the past few years: (1) preparation of nano-sized silicon materials, such as the silicon nanoparticle [14,15], silicon nanowire [16,17], silicon nanotube [18,19] and silicon film [20]. The silicon nanomaterial provides large surface area and short ion diffusion path. The characteristics of high peristalsis and high plasticity of silicon nanomaterials can alleviate the volume effect at a certain extent and improve the cycling stability of the materials. Xinliang Feng et al. used block copolymers to construct independent two-dimensional structures model [21,22]. However, the existence and conservation of two-dimensional structure is very difficult; it is easy to agglomerate and form three-dimensional (3D) large particles. Therefore, the maintenance of two-dimensional structure is of great significance for the nanocrystallization of materials. (2) Preparation of silicon composites by introduction of the second phase, such as silicon/carbon composite material [23-25] and silicon-based metal complex [26,27]. The volume change of silicon during battery cycling is suppressed by the excellent mechanical properties of the second phase, and the conductivity of the composite can also be increased because of the introduction of the second phase. Thus, the rate performance of electrode will be improved. A combination of these two strategies is expected to be a promising approach toward high-performance silicon-based anode material. However, it is difficult to realize the controllable and large-scale synthesis of nanostructured silicon/carbon composite because low cost and scalable fabrication method free of using highly reactive silane species are rare [28,29]. Dong Jin Yoo et al. prepared a doped three-dimensional/small particle composite structure by reuniting with graphene oxide and platinum particles $[30,31]$. The composite has good catalytic activity and high stability in the field of fuel cell catalysts. Therefore, the three-dimensional structure and nano-size of materials are of great significance for improving the properties of materials.

In this work, octavinyl-polyhedral oligomeric silsesquioxane is used as a silicon source and inorganic-organic crosslink agent for the synthesis of nano Si@C material. Because the polyhedral oligomeric silsesquioxane has a three-dimensional cage-like framework $[32,33]$ and its size is at the nanometer level, the thus-developed Si@C negative electrode material prepared here preserves the three-dimensional pore structure of the silicon polyoxane. Such a unique morphology can alleviate the volume expansion of silicon material well. After magnesium heat reduction treatment, a conformable carbon coating is formed on the surface of silicon nanostructures. The coated carbon can also buffer the volume changes of silicon species during the cycling. At the same time, due to the existence of the pore structure in the developed Si@C material, the carbon phase in the composite would re-organize during the charge-discharge process, thus increasing the cycle life. Finally, the Si@C negative electrode material structure change along with the charge/discharge cycles of this synthesized material is investigated in order to get the mechanism of the capacity variation upon this kind of material during the whole life of charge-discharge cycles.

\section{Materials and Methods}

Octavinyl-POSS(polyhedral oligomeric silsesquioxane), styrene and initiator AIBN (azobisisobutyronitrile) were purchased from Sigma-Aldrich Co. Ltd (Shanghai, China). Octavinyl-POSS, styrere and AIBN were added in a two-necked flask. After stirring for $10 \mathrm{~min}$, the flask was placed in liquid nitrogen. During the freeze-pump-thaw cycles, oxygen was driven away. Then, the flask was transferred into the oil bath with mild magnetic stirring at $65{ }^{\circ} \mathrm{C}$ under nitrogen atmosphere. When the solution became transparent gel, copolymerization reaction finished. The production was vacuum dried at $80^{\circ} \mathrm{C}$ for $9 \mathrm{~h}$. After that, the poly(POSS-styrene) was carbonized 
at $900{ }^{\circ} \mathrm{C}$ for $3 \mathrm{~h}$ under the protection of Ar with a heating rate of $3{ }^{\circ} \mathrm{C} \mathrm{min}-1$. The as-obtained carbonized product was treated with magnesiothermic reduction at $650{ }^{\circ} \mathrm{C}$ for $2 \mathrm{~h}$ under the protection of Ar. The end-product was washed with $1 \mathrm{~mol} / \mathrm{L} \mathrm{HCl}$ and distilled water for several times and dried at $80{ }^{\circ} \mathrm{C}$ for $12 \mathrm{~h}$. The Si@C anode material was then obtained. For comparison, the carbon material without $\mathrm{Si}$ was also prepared by etching the Si@C with analytic grade HF for $3 \mathrm{~h}$, followed by repeated washing with ethanol six times.

The Si@C material was mixed with acetylene black and poly(vinylidene fluoride) (PVDF) to form a slurry at a mass ratio of 7:2:1 in N-Methyl pyrrolidone (NMP). After stirring for $12 \mathrm{~h}$, the slurry was coated on copper foil by using the 90um spreader. Then, the slurry was dried at $70{ }^{\circ} \mathrm{C}$ for $12 \mathrm{~h}$. The Si@C anode was then incorporated into coin cell in an argon filled glove box with a lithium foil as counter electrode, a Celgard separator and $1 \mathrm{M} \mathrm{LiPF}_{6}$ in a 1:1 ethyl carbonate (EC): dimethyl carbonate (DMC) solvent as electrolyte.

All the electrochemical tests were accomplished at room temperature. The galvanostatic charge-discharge tests were carried out using a Land CT2001A (whshland Co. Ltd., Wuhan, China) between $0.001-3 \mathrm{~V}$. The cyclic voltammetry (CV) tests were carried out between $0.001-3 \mathrm{~V}$ by using a CHI660D electrochemical workstation (CH Instruments Co. Ltd., Shanghai, China) with a scan rate of $0.1 \mathrm{mVs}^{-1}$. And the electrochemical impedance spectroscopy (EIS) tests were carried out between $0.001 \mathrm{~Hz}-10 \mathrm{KHz}$ by using CorrTest CS310 electrochemical workstation (Wuhan Corrtest Instrument Co. Ltd., Wuhan, China) and the AC signal amplitude was $5 \mathrm{mV}$.

The material morphology and EDS mapping were observed by the scanning electron microscope (SEM, JSM-IT300) and the transmission electron microscope (TEM, Talos F200S, FEI, Waltham, MA, USA). The material structure was performed by the X-ray diffractometer (XRD, D8 Advance), the RENISHAW Raman microscope (Raman, RENISHAW InVia, Wotton-under-Edge, UK) and the X-ray photoelectron spectroscopy (XPS, ESCALAB 250Xi, Thermo Fisher Scientific, Waltham, MA, USA).

\section{Results}

Figure 1a shows the synthesis process for the POSS-derived Si@C anode material. In this synthesis, octavinyl-POSS, as an organic-inorganic silicon source, introduces polyhedral silicon framework and nanoscale pores to the material system. Styrene is selected as a carbon source and a cross-linker to form 3D copolymer network as shown in Figure 1b. The formation of copolymer is based on copolymerization of vinyl monomers with a thermal initiator. After high temperature carbonization and magnesium reduction of the copolymer, carbon coated silicon species ( $\mathrm{Si} @ \mathrm{C}$ ) will be formed. The macroscopic morphology of the polymerized gel product and the final carbonized product are shown in Figure 1c.

The poly (POSS-styrene) retains the 3D skeleton of octavinyl-POSS well, as shown in Figure 2a. The magnified SEM image of the poly(POSS-styrene) is shown in Figure $2 \mathrm{~b}$. After carbonization and reduction, the product exhibits a typical loose porous feature (Figure 2c). Element mapping of the final products reveals that carbon and silicon are evenly distributed in the sample (Figure 2d). Figure S1a,b (supplementary material) show XRD and Raman of POSS monomer and the polymer. The peak position of the polymer is roughly the same as the peak position of the monomer, indicating that the polymerization does not affect the crystal structure of POSS.

Several broadening peaks are detected by the XRD measurement in Figure 2e, confirming the low degree of graphitization of carbon skeleton in Si@C. The XRD pattern shows that the magnesiothermic reduction process can promote the formation of $\mathrm{Si} @ \mathrm{C}$ composite structure. The broad peak around $25^{\circ}$ can be attributed to $\mathrm{MHSiO}_{2}$ in amorphous, and another wide peak at $40^{\circ}$ can be attributed to $\mathrm{MHSiO}_{2}$ complexed with amorphous carbon $[34,35]$. Raman in Figure 2f shows the broadening silicon peak at $400-500 \mathrm{~cm}^{-1}$ and $800-900 \mathrm{~cm}^{-1}$ [36]. In these Raman spectra, the D-band is located at $1340 \mathrm{~cm}^{-1}$ to characterize the defects of carbon atomic crystals and the G-band is located at $1556 \mathrm{~cm}^{-1}$ to characterize 
the plane expansion vibration of carbon atom SP2 hybrid $[37,38]$. The intensity ratio $\mathrm{I}_{\mathrm{D}} / \mathrm{I}_{\mathrm{G}}$ close to 1 further shows the low degree of graphitization of Si@C anode material.

(a)
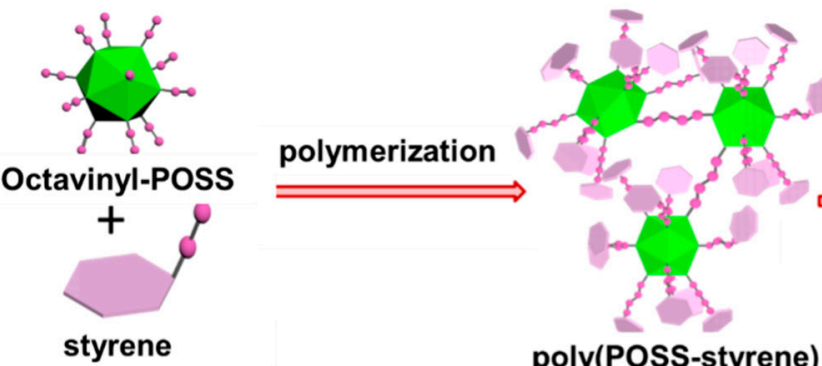

(b)
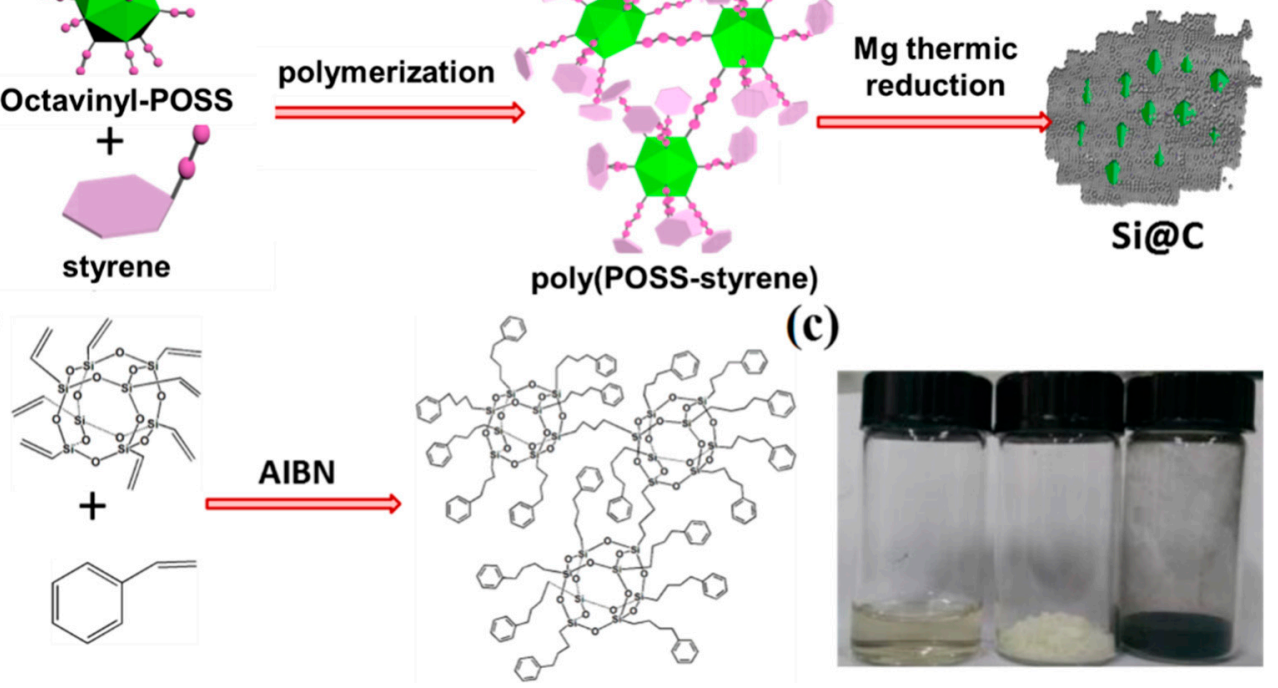

(c)

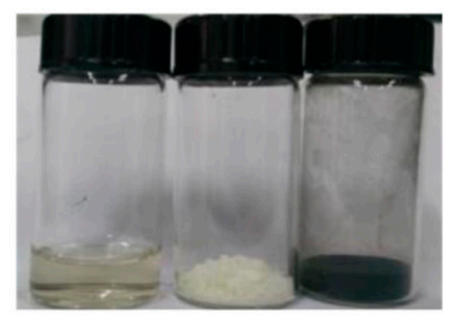

Figure 1. (a) Schematic illustration of synthesis of polyhedral oligomeric silsesquioxane (POSS)-derived $\mathrm{Si} @ \mathrm{C}$ anode material. (b) Formation of poly(POSS-styrene) between Octavinyl-POSS and styrene cross-linker via Vinyl copolymerization. (c) The photos of poly(POSS-styrene), dry-poly(POSS-styrene) and Si@C.
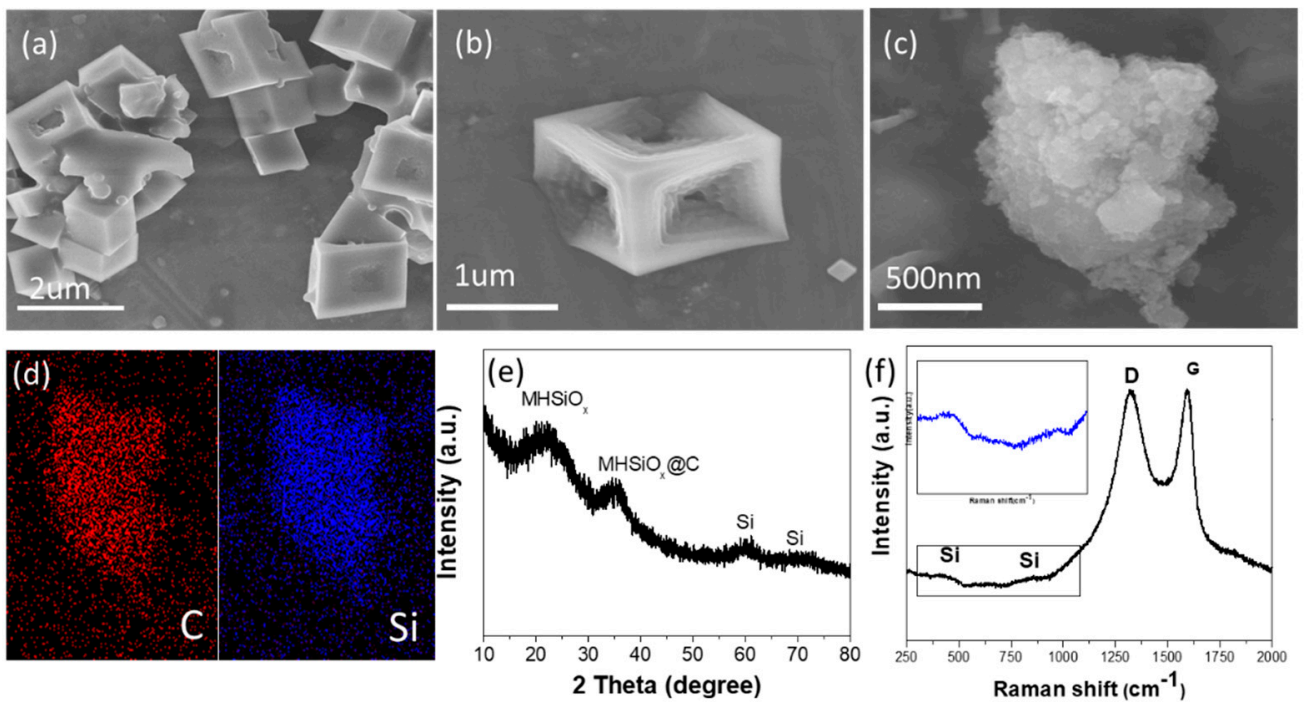

Figure 2. (a,b) Scanning electron microscope (SEM) images of poly(POSS-styrene) at different magnification. (c,d) SEM image and elemental mapping of Si@C. (e) X-ray diffractometer (XRD) patterns of Si@C. (f) Raman spectra of Si@C.

The TEM image and elemental mapping of POSS monomer in Figure 3a show the octahedron morphology, confirm that the distribution of element in monomer is uniform. According to the area of elemental mapping of poly (POSS-styrene) in Figure 3b, carbon coats on the silicon surface after cross-linking and the integrity of the silicon skeleton well preserves. Figure $3 \mathrm{c}$ shows the morphology and elemental distribution of Si@C. It illustrates that even if the silicon skeleton collapses, silicon is still coated with carbon. This characteristic can help to buffer the expansion of silicon during charging and discharging. 

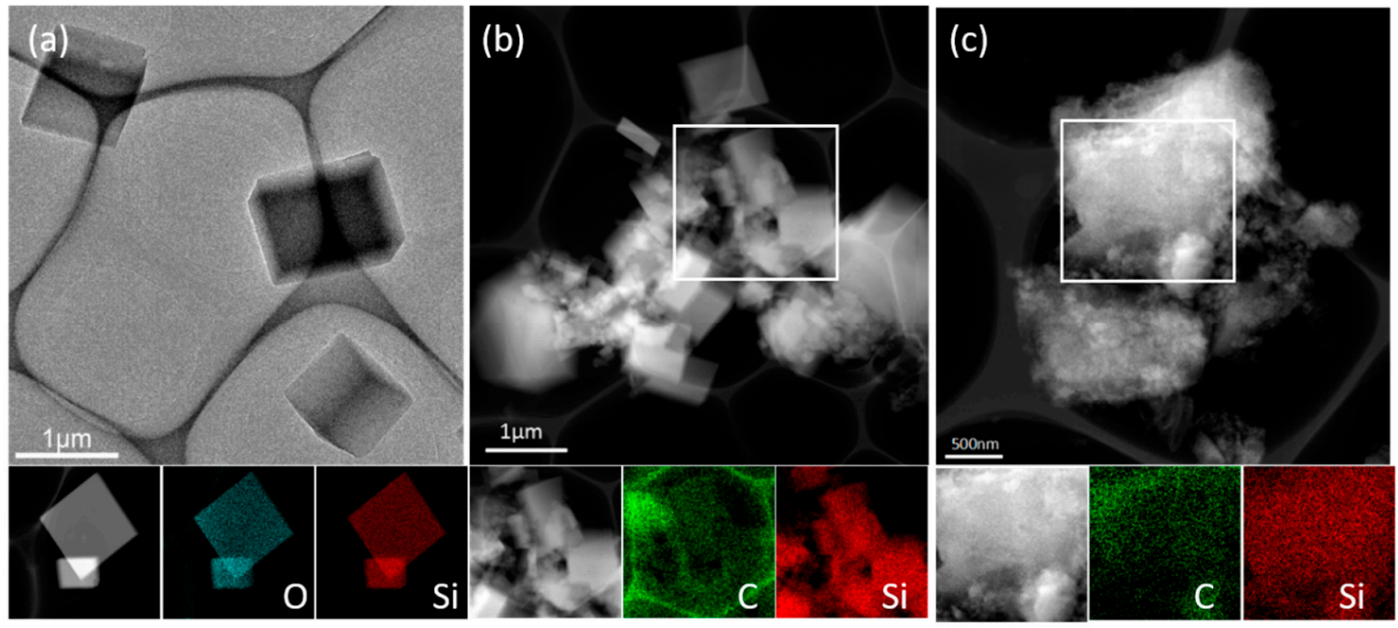

Figure 3. Transmission electron microscope (TEM) image and an area elemental mapping of poss (a), polymer (b) and Si@C (c).

The chemical states of POSS, Polymer and Si@C materials are analyzed by XPS. The accurate silicon content within the Si@C, determined by atomic absorption spectroscope, is $39.27 \mathrm{wt} . \%$. As shown in Figure 4a, the full-range XPS surveys present the existence of silicon, carbon and oxygen. The high-resolution spectra of Si $2 p$ and C 1s are shown in Figure $4 b$,c. After copolymerization, the peak position of $\mathrm{SiOx}$ located at $102.6 \mathrm{eV}$ ) shifts between POSS and polymer [39]. The $\mathrm{Si} 2 \mathrm{p}$ spectra of Si@C exhibits four peaks located at 100.4, 102.6, 102.8 and $103.6 \mathrm{eV}$, which correspond to Si-C, $\mathrm{Si}^{2+}, \mathrm{Si}^{3+}$ and $\mathrm{Si}^{4+}$, respectively. The presence of these $\mathrm{Si}$ species demonstrates the multiple oxidation states of $\mathrm{Si}$ in the composite and carbon phase is in close vicinity of Si species after carbonization and magnesiothermic reduction. In Figure $4 \mathrm{~b}$, to compare the spectra of $\mathrm{C} 1 \mathrm{~s}$ between POSS and Polymer, the peak located at 284.5 and $284.6 \mathrm{eV}$ corresponding to $\mathrm{C}=\mathrm{C}$ and $\mathrm{C}-\mathrm{C}$ indicates the copolymerization of vinyl radical. The spectra of C 1s of Si@C shows the peak of C-Si located at $283.5 \mathrm{eV}$, which is consistent with the peak of Si-C located at $100.4 \mathrm{eV}$.
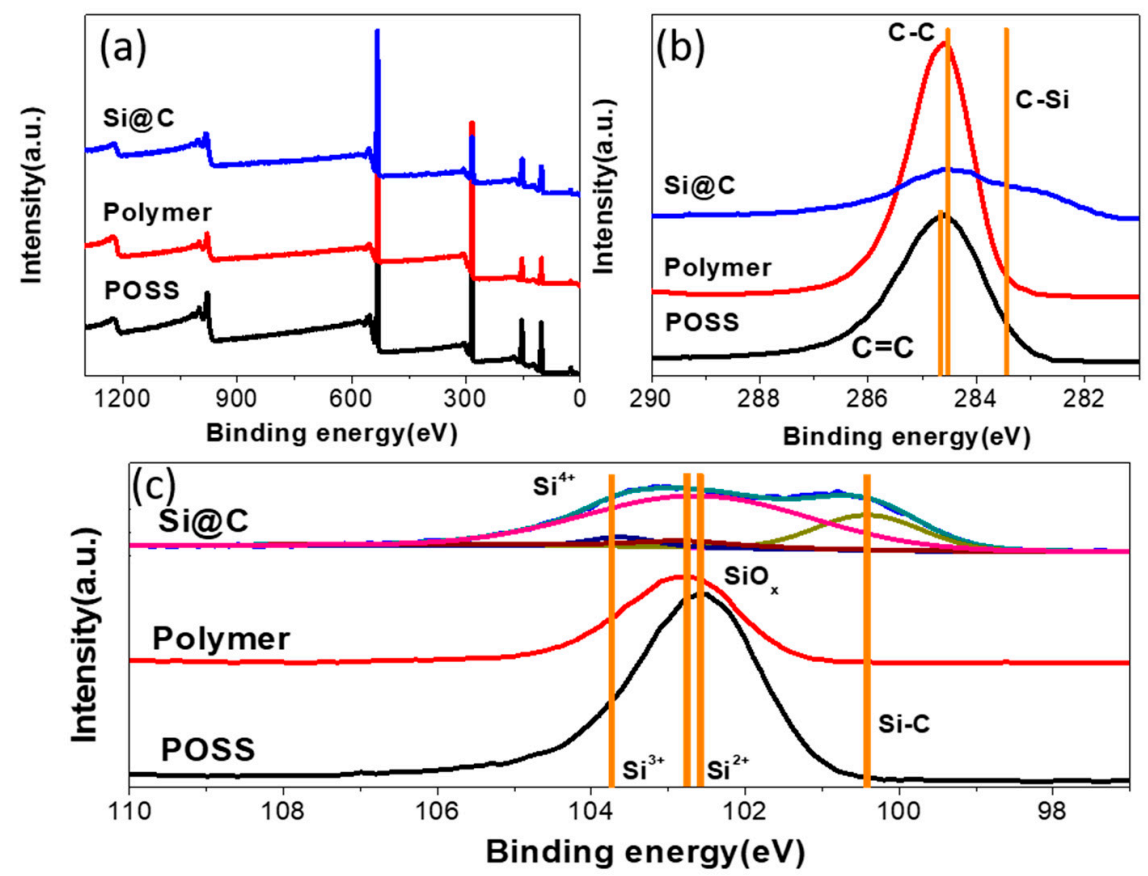

Figure 4. (a) X-ray photoelectron spectroscopy (XPS) survey spectra, and XPS spectra for the (b) C 1s and Si $2 \mathrm{p}(\mathrm{c})$ of POSS, polymer and Si@C. 
Electrochemical characteristics of synthesized carbon material without $\mathrm{Si}$ are shown in Figure 5. In the first three CV curves, as shown in Figure 5a, the redox peaks and potential plateaus are not obvious. As shown in Figure 5b, the capacity of the battery below $0.5 \mathrm{~V}$ was mainly contributed by lithium insertion into the graphitic layers. The appearance of a potential slope indicates the disordered stacking of the graphitic layers, which leads to electrochemically and geometrically nonequivalent active lithium sites [40,41]. The first cycle discharge capacity of synthesized carbon material without $\mathrm{Si}$ is about $1250 \mathrm{mAh} \mathrm{g}^{-1}$. After a couple of cycles, the discharge capacity attenuates drastically to $380 \mathrm{mAh} \mathrm{g}^{-1}$ (in Figure 5b,c).
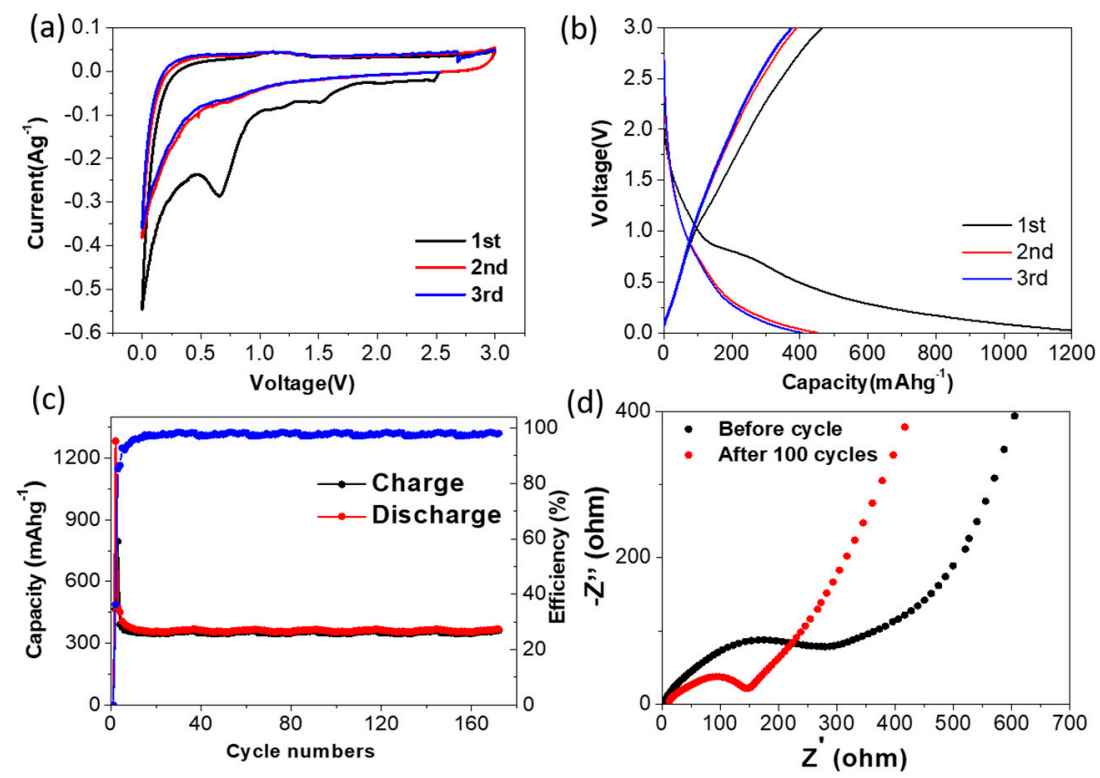

Figure 5. (a) First three CV curves of synthesized carbon material without $\mathrm{Si}$ at a scan rate of $0.1 \mathrm{mVs}^{-1}$. (b) First three galvanostatic charge-discharge curves of synthesized carbon material without Si. (c) Cycling performance of synthesized carbon material without $\mathrm{Si}$ at a rate of $200 \mathrm{mAh} \mathrm{g}^{-1}$. (d) Electrochemical impedance spectra of synthesized carbon material without Si before and after cycling.

The electrochemical characteristics of Si@C are shown in Figure 6. During the CV and charge-discharge curves (Figure 6a,b), the broad reduction peak (about $1.5 \mathrm{~V}$ ) confirms the preliminary decomposition of the electrolyte and the formation of solid electrolyte interface (SEI) films. At about $1.3 \mathrm{~V}$, the broad oxidation peaks are observed in the first three anodic scans, illustrating the reversible oxidation of some SEI components. At a charge-discharge rate of $200 \mathrm{~mA} \mathrm{~g}^{-1}$, the first cycle discharge and charge capacity of the Si@C electrode is $580 \mathrm{mAh} \mathrm{g}^{-1}$ and $1500 \mathrm{Ahg}^{-1}$, respectively as shown in Figure 6c. The large irreversible capacity exists here because of the formation of the solid electrolyte interphase (SEI) and some irreversible reactions upon the electrode and/or electrolyte [42]. There is an interesting phenomenon during charge-discharge process that the $\mathrm{Si}-\mathrm{C}$ anode undergoes through a quick capacity drop-off at initial several cycles, and then the capacity creep up is observed along subsequent cycles; Finally, as the cycle continues, the capacity reaches the initial capacity (Figure 6c). Furthermore, the Si@C anode shows the superior rate performance as shown in Figure 6c. For instance, the Si@C anode presents relatively high reversible capacities of $570 \mathrm{mAh} \mathrm{g}^{-1}, 500 \mathrm{mAh} \mathrm{g}^{-1}$ and $450 \mathrm{mAh} \mathrm{g}^{-1}$ at the rate of $1 \mathrm{~A} \mathrm{~g}^{-1}, 2 \mathrm{~A} \mathrm{~g}^{-1}$ and $5 \mathrm{~A} \mathrm{~g}^{-1}$, respectively. When the current density goes back to $200 \mathrm{~mA} \mathrm{~g}^{-1}$, the reversible capacity can recover back to the initial value of $780 \mathrm{mAh} \mathrm{g}^{-1}$. The possible reason to the excellent rate performance of Si@C anode could be that its hollow cage structure offers a better electrode-electrolyte contact for the fast transmit of $\mathrm{Li}^{+}$into $\mathrm{Si} @ \mathrm{C}$ material. In order to explore the reasons for the capacity change during the cycling, four representative analysis points were selected (number Si@C-@1, Si@C-@2, Si@C-@3 and Si@C-@4 shown in Figure 6c) and 
investigated for the mechanistic discussion. The Si@C-@1 point represents the fresh battery without cycling. The Si@C-@2 represents the battery after 20 cycles when the capacity reaches the lowest in the full cycle life. The Si@C-@3 represents the battery after 550 cycles when the capacity is the highest in the full cycle life. The Si@C-@4 represents the end point of the full cycle life.
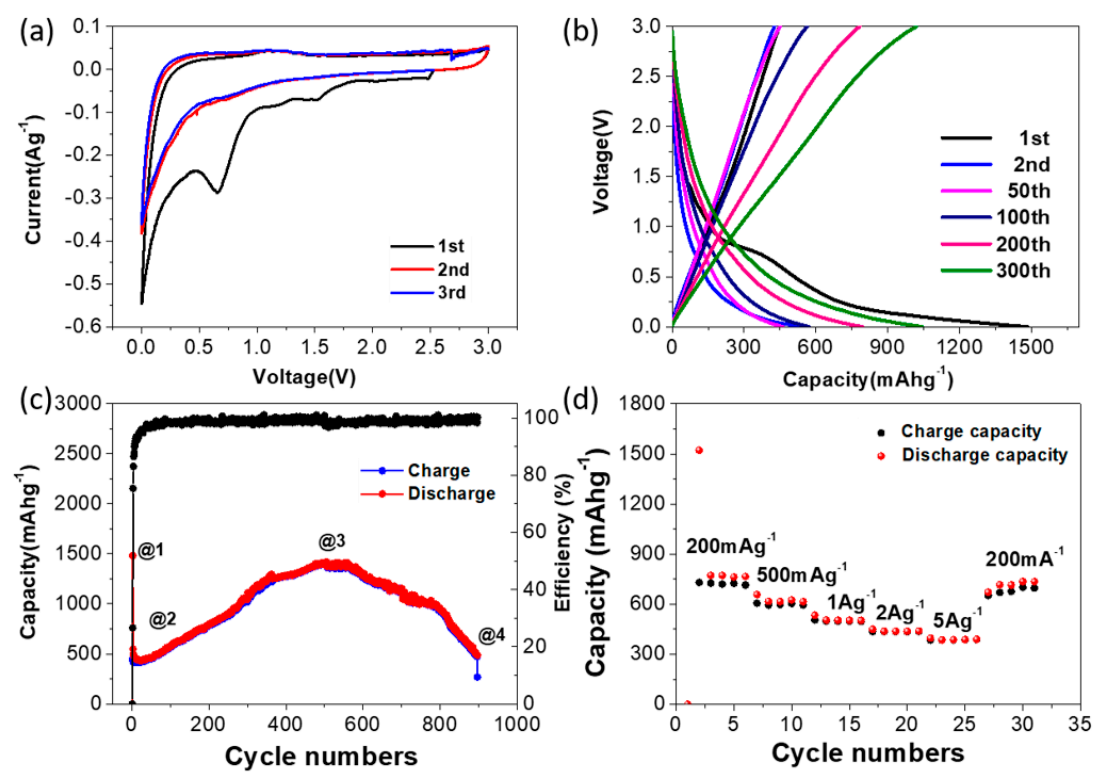

Figure 6. (a) First three CV curves of $\mathrm{Si@C}$ at a scan rate of $0.1 \mathrm{mVs}^{-1}$. (b) Galvanostatic charge-discharge curves of Si@C at a rate of $200 \mathrm{mAg}^{-1}$. (c) Full life cycling performance of Si@C at a rate of $200 \mathrm{mAg}^{-1}$. (d) Rate performance of Si@C at different rates.

Electrochemical impedance measurements are carried out to discuss the electrode kinetic information of Si@C anode at these four analysis points (Figure 7). The Nyquist plot includes three parts: (1) a semicircle at high frequency region which characterizes partial de-solvation and adsorption of lithium ions onto the surface of the electrode; (2) a semicircle at intermediate frequency region representing the desolvated lithium ions into the lattice of active materials; (3) an oblique line at low frequency region relating to the solid-state diffusion of lithium ions [43-45]. In general, the electrode process is mainly controlled by the charge transfer and diffusion processes and the Nyquist diagram is composed of the semicircle in the high frequency region and the sloped line of the low frequency region. The resistance $R_{c t}$ represents the charge transfer resistance of the electrochemical reaction and the charge transfer resistance has a great relationship with the electrochemical activity of the electrode material. The resistance $R_{\mathrm{ct}}$ of Si@C-@3 $(\sim 40 \Omega)$ is minimum that may indicate the maximum activation of silicon and mutual embedment between silicon and carbon. The $\mathrm{R}_{\mathrm{ct}}$ of Si@C-@2 $(\sim 300 \Omega)$ is significantly larger than in the Si@C-@1 $(\sim 100 \Omega)$ that illustrates the formation of solid electrolyte interphase (SEI) and the initial irreversible reaction during initial charge-discharge cycles. The resistance $\mathrm{R}_{\mathrm{ct}}$ of Si@C-@4 $(\sim 500 \Omega)$ is maximum that may indicate the structural collapse and the pulverization of grain after long-term charge-discharge. The obvious reduced resistance between Si@C-@2 and Si@C-@3 is beneficial for promoting the transport and storage of lithium ions, which explains the increased lithium storage capacity for as-obtained Si@C anode from 20 cycles to 550 cycles. The phenomenon of the increase on capacity after cycling had been reported for carbonaceous material. However, few reports explain the cause and mechanism of the rise of the capacity [46-48].

Furthermore, four representative cells cycled to the desired analysis points (in the complete delithiation condition) were disassembled in the glove box and Si@C anode material in these cells were characterized to further explain the mechanism for the capacity variation. Figure 8 shows the morphology and element distribution of the Si@C anode material in these four analysis points. Compared with Si@C-@1 (Figure 8), fluorine can be observed in Si@C-@2 (Figure 8). This result 
suggests that during the first charge and discharge process, the electrolyte diffuses into the sample to form a solid electrolyte membrane, causing an irreversible capacity change, which agrees with the rapid decline of the capacity at this time. In Si@C-@3 (Figure 8), the silicon surface is coated with a dense layer of carbon compared to Si@C-@1 and Si@C-@2. During the following cycles, carbon gradually penetrates into the silicon frame structure, relieves the volume expansion caused by the silicon itself in the process of charging and discharging and enhances the cyclic stability of the battery. At the same time, the continuous activation of silicon increases the lithium intercalation site and increases the lithium storage capacity, resulting in a capacity up to $1430 \mathrm{mAh} \mathrm{g}^{-1}$. In Figure 8, after long-term charge-discharge cycling, most of the carbon has been separated from the silicon surface and the structure collapsed, leading to a decline of capacity in later period.

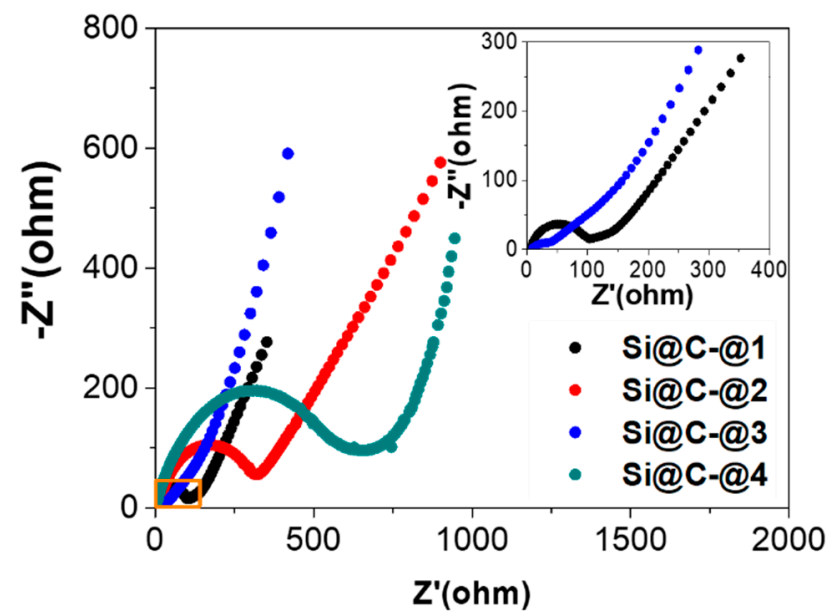

Figure 7. Electrochemical impedance spectra obtained at Si@C-@1, Si@C-@2, Si@C-@3 and Si@C-@4 points.

(a)

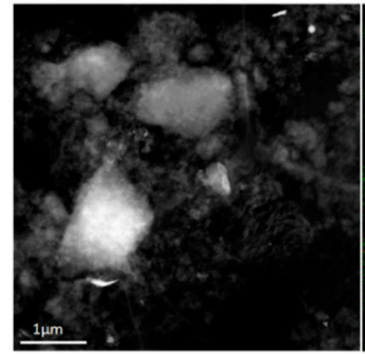

(b)

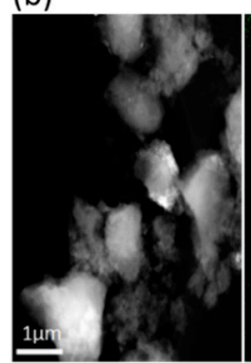

(c)

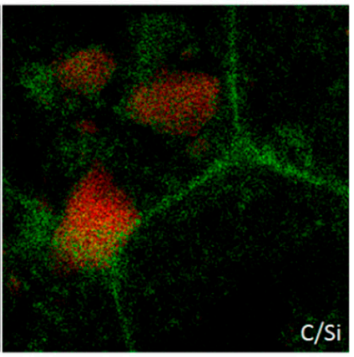

c/si

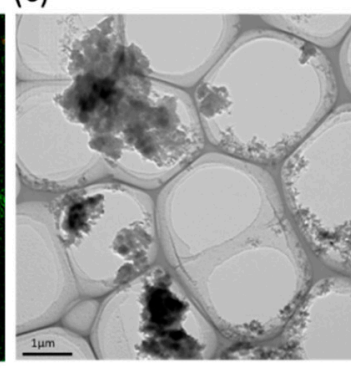

(d)

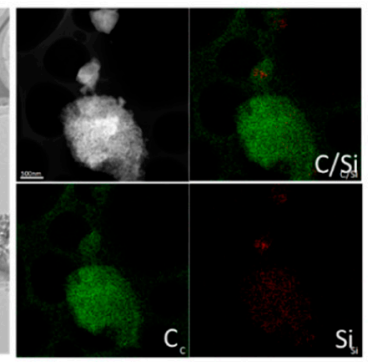

$\mathrm{Si}$

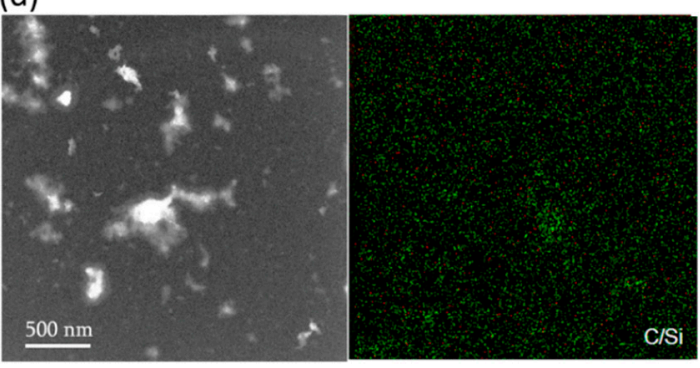

Figure 8. TEM image and elemental mapping of (a) Si@C-@1, (b) Si@C-@2, (c) Si@C-@3 and (d) Si@C-@4.

In Figure 9, by analyzing the composition of Si@C anode material in the four analysis points, the mechanism of capacity variation can be further unveiled. According to the small angle X-ray diffraction in Figure 9a, the peak of silicon ( 46 $)$ in Si@C-@4 is clearly observed due to the pulverization and abscission of silicon particles after a long-term charge and discharge. In the XPS survey spectra 
(Figure 9b), it is observed that the F 1s peak intensity in Si@C-@2 is the highest within four analysis points, indicating that the effect of the solid electrolyte membrane is dominant in Si@C-@2, which can explain the sharp capacity decline in the first few charge and discharge cycles. In $\mathrm{Si} 2 \mathrm{p}$ spectra (Figure 9c), compared to Si@C-@2, the chemical state of silicon in Si@C-@3 is mainly the chemical bond with carbon, indicates a conformable carbon coating on silicon, agreeing well the distribution of elements shown in Figure 9c. In Si@C-@4, the Si 2p spectra are dominated by silicon-silicon bonds. The similar trend can be also observed in XPS spectra for the C 1s (Figure 9d). Compared with Si@C-@3 and Si@C-@4, the intensity of Carbon-Fluorine peak is found to be the strongest in Si@C-@2. In Si@C-@4, the disappearance of the carbon-silicon peak matches well with the XPS spectra of the Si2p in the Si@C-@4 (Figure 9c). And long charge-discharge cycles, the electrolyte will decompose and volatilize to produce free $\mathrm{HF}$, leading to insufficient electrolyte for complete charge-discharge reaction, thus resulting in the decline of the actual capacity. The appearance of free HF can damage the anode material and metal ion $(\mathrm{Al}, \mathrm{Mg}, \mathrm{Ba}, \mathrm{Li}, \mathrm{Ca})$ in electrolyte can eliminate the free $\mathrm{HF}$ from the decomposition of $\mathrm{LiPF}_{6}$. The peak of the C-M appeared in Si@C-@4, indicating the destroying of the charge-discharge system.
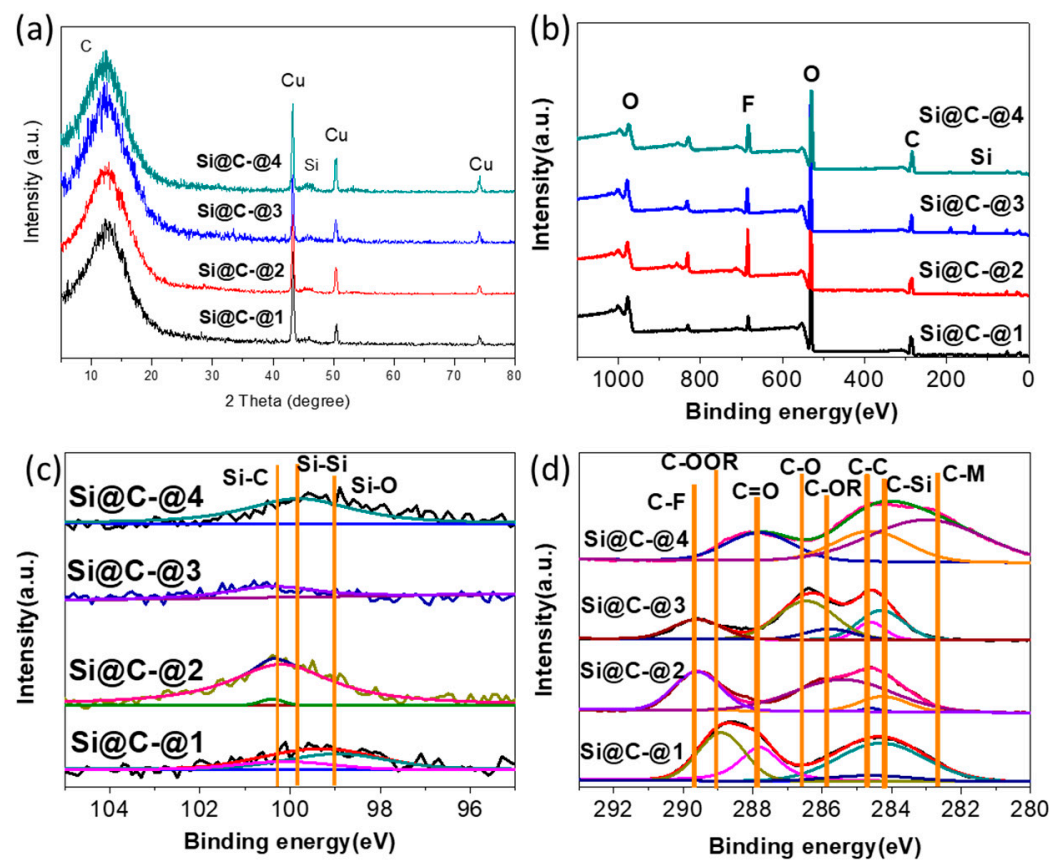

Figure 9. (a) XRD patterns of Si@C-@1, Si@C-@2, Si@C-@3 and Si@C-@4. (b) XPS survey spectra for Si@C-@1, Si@C-@2, Si@C-@3 and Si@C-@4. XPS spectra for the (c) Si 2p and (d) C 1s of Si@C-@1, Si@C-@2, Si@C-@3 and Si@C-@4. (M represents alkali metal or alkaline earth metal existed in the lithium salt and electrolyte additives.).

\section{Conclusions}

In summary, POSS-derived Si@C was developed by carbonization of the precursor, followed by magnesium thermal reduction. As an LIBs anode material, it shows a good performance of lithium storage performance due to its unique nanostructure. The half battery with Si@C anode delivered an initial discharge capacity up to $1500 \mathrm{mAh} \mathrm{g}^{-1}$ and a high discharge capacity of $1430 \mathrm{mAh} \mathrm{g}^{-1}$ was retained after 550 cycles. In addition, we observed a rapid fade in discharge capacity of the electrode after 550 cycles. To understand such a discharge behavior, a combined analysis of the electrode with XRD, XPS, TEM and ex-situ impedance measurements were performed with Si@C anode at different cycling states. Our results show that the change in the discharge capacity is closely related to the changes of electrode composition induced by the electrolyte decomposition, agreeing with previous finds that the electrochemical properties of an electrode is closely related to the nanostructure of the 
material [49]. Our results demonstrate that the novel Si@C material is a promising alternative to existing graphite anode. In addition, the mechanism for the capacity change revealed in this study may provide useful guidance for future development of electrode materials.

Supplementary Materials: The following are available online at http:/ /www.mdpi.com/2073-4360/11/4/576/s1, Figure S1: XRD and Raman spectra of POSS, Figure S2: electrochemical performance of the electrode.

Author Contributions: Conceptualization, M.T. and H.T.; methodology, Z.B., J.Z., Z.D. and D.L.; analysis, Z.B., J.Z., J.L, Z.D. and D.L.; investigation, D.L.; analysis, Z.B., J.Z., J.L., Z.D. and D.L.; writing-original draft preparation, Z.B.; writing-review and editing, J.L.

Funding: This research was funded by the National Natural Science Foundation of China (51472187), Fundamental Research Funds for the Central Universities (WUT: 2018-IB-026) and the State Key Laboratory of Advanced Technology for Material Synthesis and Processing (Wuhan University of Technology, 2019-KF-10).

Conflicts of Interest: The authors declare no conflict of interest.

\section{References}

1. Vinothkannan, M.; Kim, A.R.; Kumar, G.G.; Yoo, D.J. Sulfonated graphene oxide/Nafion composite membranes for high temperature and low humidity proton exchange membrane fuel cells. RSC Adv. 2018, 8, 7494-7508. [CrossRef]

2. Vinothkannan, M.; Kim, A.R.; Kumar, G.G.; Yoon, J.-M.; Yoo, D.J. Toward improved mechanical strength, oxidative stability and proton conductivity of an aligned quadratic hybrid (SPEEK/FPAPB/Fe 3 O 4 -FGO) membrane for application in high temperature and low humidity fuel cells. RSC Adv. 2017, 7, 39034-39048. [CrossRef]

3. Choi, J.W.; Aurbach, D. Promise and reality of post-lithium-ion batteries with high energy densities. Nat. Rev. Mater. 2016, 1, 16013. [CrossRef]

4. Lewis, N.S. Research opportunities to advance solar energy utilization. Science 2016, 351. [CrossRef] [PubMed]

5. Cui, Q.; Zhong, Y.; Pan, L.; Zhang, H.; Yang, Y.; Liu, D.; Teng, F.; Bando, Y.; Yao, J.; Wang, X. Recent Advances in Designing High-Capacity Anode Nanomaterials for Li-Ion Batteries and Their Atomic-Scale Storage Mechanism Studies. Adv. Sci. 2018, 5, 1700902. [CrossRef] [PubMed]

6. Chu, Q.; Yang, B.; Tong, W.; Wang, X.; Liu, X.; Chen, J.; Wang, W. Fabrication of a Stainless-Steel-Mesh-Supported Hierarchical Fe 2 O 3 @NiCo 2 O 4 Core-Shell Tubular Array Anode for Lithium-Ion Battery. ChemistrySelect 2016, 1, 5569-5573. [CrossRef]

7. Ma, N.; Jiang, X.-Y.; Zhang, L.; Wang, X.-S.; Cao, Y.-L.; Zhang, X.-Z. Novel 2D Layered Molybdenum Ditelluride Encapsulated in Few-Layer Graphene as High-Performance Anode for Lithium-Ion Batteries. Small 2018, 14, 1703680. [CrossRef]

8. Tang, Q.; Su, H.; Cui, Y.; Baker, A.P.; Liu, Y.; Lu, J.; Song, X.; Zhang, H.; Wu, J.; Yu, H.; et al. Ternary tin-based chalcogenide nanoplates as a promising anode material for lithium-ion batteries. J. Sources 2018, 379, 182-190. [CrossRef]

9. Wu, Y.; Huang, L.; Huang, X.; Guo, X.; Liu, D.; Zheng, D.; Zhang, X.; Ren, R.; Qu, D.; Chen, J. A room-temperature liquid metal-based self-healing anode for lithium-ion batteries with an ultra-long cycle life. Environ. Sci. 2017, 10, 1854-1861. [CrossRef]

10. Zuo, X.; Zhu, J.; Müller-Buschbaum, P.; Cheng, Y.-J. Silicon based lithium-ion battery anodes: A chronicle perspective review. Nano Energy 2017, 31, 113-143. [CrossRef]

11. Shen, X.; Tian, Z.; Fan, R.; Shao, L.; Zhang, D.; Cao, G.; Kou, L.; Bai, Y. Research progress on silicon/carbon composite anode materials for lithium-ion battery. J. Chem. 2018, 27, 1067-1090. [CrossRef]

12. Hertzberg, B.; Alexeev, A.; Yushin, G. Deformations in Si-Li Anodes Upon Electrochemical Alloying in Nano-Confined Space. J. Am. Chem. Soc. 2010, 132, 8548-8549. [CrossRef]

13. An, S.J.; Li, J.; Daniel, C.; Mohanty, D.; Nagpure, S.; Wood, D.L.; Iii, D.L.W. The state of understanding of the lithium-ion-battery graphite solid electrolyte interphase (SEI) and its relationship to formation cycling. Carbon 2016, 105, 52-76. [CrossRef]

14. Kovalenko, I.; Zdyrko, B.; Magasinski, A.; Hertzberg, B.; Milicev, Z.; Burtovyy, R.; Luzinov, I.; Yushin, G. A Major Constituent of Brown Algae for Use in High-Capacity Li-Ion Batteries. Science 2011, 334, 75-79. [CrossRef] 
15. Haro, M.; Singh, V.; Steinhauer, S.; Toulkeridou, E.; Grammatikopoulos, P.; Sowwan, M. Nanoscale Heterogeneity of Multilayered Si Anodes with Embedded Nanoparticle Scaffolds for Li-Ion Batteries. Adv. Sci. 2017, 4, 1700180. [CrossRef]

16. Salvatierra, R.V.; Raji, A.-R.O.; Lee, S.-K.; Ji, Y.; Li, L.; Tour, J.M. Silicon Nanowires and Lithium Cobalt Oxide Nanowires in Graphene Nanoribbon Papers for Full Lithium Ion Battery. Adv. Mater. 2016, 6, 1600918. [CrossRef]

17. Song, H.; Wang, S.; Song, X.; Yang, H.; Du, G.; Yu, L.; Xu, J.; He, P.; Zhou, H.; Chen, K.J. A bottom-up synthetic hierarchical buffer structure of copper silicon nanowire hybrids as ultra-stable and high-rate lithium-ion battery anodes. J. Mater. Chem. A 2018, 6, 7877-7886. [CrossRef]

18. Kim, Y.-Y.; Kim, H.-J.; Jeong, J.-H.; Lee, J.; Choi, J.-H.; Jung, J.-Y.; Lee, J.-H.; Cheng, H.; Lee, K.-W.; Choi, D.-G.; et al. Facile Fabrication of Silicon Nanotube Arrays and Their Application in Lithium-Ion Batteries. Adv. Eng. Mater. 2016, 18, 1349-1353. [CrossRef]

19. Wang, W.; Gu, L.; Qian, H.; Zhao, M.; Ding, X.; Peng, X.; Sha, J.; Wang, Y. Carbon-coated silicon nanotube arrays on carbon cloth as a hybrid anode for lithium-ion batteries. J. Sources 2016, 307, 410-415. [CrossRef]

20. Suresh, S.; Wu, Z.P.; Bartolucci, S.F.; Basu, S.; Mukherjee, R.; Gupta, T.; Hundekar, P.; Shi, Y.; Lu, T.-M.; Koratkar, N. Protecting Silicon Film Anodes in Lithium-Ion Batteries Using an Atomically Thin Graphene Drape. ACS Nano 2017, 11, 5051-5061. [CrossRef] [PubMed]

21. Liu, S.; Zhang, J.; Dong, R.; Gordiichuk, P.; Zhang, T.; Zhuang, X.-D.; Mai, Y.; Liu, F.; Herrmann, A.; Feng, X. Two-Dimensional Mesoscale-Ordered Conducting Polymers. Angew. Chem. Int. Ed. 2016, 55, 12516-12521. [CrossRef] [PubMed]

22. Liu, S.; Gordiichuk, P.; Wu, Z.-S.; Liu, Z.; Wei, W.; Wagner, M.; Mohamed-Noriega, N.; Wu, D.; Mai, Y.; Herrmann, A.; et al. Patterning two-dimensional free-standing surfaces with mesoporous conducting polymers. Nat. Commun. 2015, 6, 8817. [CrossRef] [PubMed]

23. Feng, K.; Li, M.; Liu, W.; Kashkooli, A.G.; Xiao, X.; Cai, M.; Chen, Z. Silicon-Based Anodes for Lithium-Ion Batteries: From Fundamentals to Practical Applications. Small 2018, 14, 1702737. [CrossRef]

24. Long, W.; Fang, B.; Ignaszak, A.; Wu, Z.; Wang, Y.-J.; Wilkinson, D. Biomass-derived nanostructured carbons and their composites as anode materials for lithium ion batteries. Chem. Soc. Rev. 2017, 46, 7176-7190. [CrossRef] [PubMed]

25. Jiang, Y.; Zhang, Y.; Yan, X.; Tian, M.; Xiao, W.; Tang, H. A sustainable route from fly ash to silicon nanorods for high performance lithium ion batteries. Chem. Eng. J. 2017, 330, 1052-1059. [CrossRef]

26. Fukata, N.; Mitome, M.; Bando, Y.; Wu, W.; Wang, Z.L. Lithium ion battery anodes using Si-Fe based nanocomposite structures. Nano Energy 2016, 26, 37-42. [CrossRef]

27. Park, H.; Choi, S.; Lee, S.-J.; Cho, Y.-G.; Hwang, G.; Song, H.-K.; Choi, N.-S.; Park, S. Design of an ultra-durable silicon-based battery anode material with exceptional high-temperature cycling stability. Nano Energy 2016, 26, 192-199. [CrossRef]

28. Chen, X.; Li, X.; Ding, F.; Xu, W.; Xiao, J.; Cao, Y.; Meduri, P.; Liu, J.; Graff, G.L.; Zhang, J.-G. Conductive Rigid Skeleton Supported Silicon as High-Performance Li-Ion Battery Anodes. Nano Lett. 2012, 12, 4124-4130. [CrossRef]

29. Li, X.; Tian, X.; Yang, T.; Wang, W.; Song, Y.; Guo, Q.; Liu, Z. Silylated functionalized silicon-based composite as anode with excellent cyclic performance for lithium-ion battery. J. Sources 2018, 385, 84-90. [CrossRef]

30. Ramakrishnan, S.; Karuppannan, M.; Vinothkannan, M.; Ramachandran, K.; Kwon, O.J.; Yoo, D.J. Ultrafine Pt Nanoparticles Stabilized by MoS2/N-Doped Reduced Graphene Oxide as a Durable Electrocatalyst for Alcohol Oxidation and Oxygen Reduction Reactions. ACS Appl. Mater. Interfaces 2019. [CrossRef]

31. Ravi, A.; Mohanraj, V.; Rhan, K.A.; Jin, Y.D. Cumulative effect of bimetallic alloy, conductive polymer and graphene toward electrooxidation of methanol: An efficient anode catalyst for direct methanol fuel cells. J. Alloys Compd. 2019, 771, 477-488.

32. Liu, D.; Cheng, G.; Zhao, H.; Zeng, C.; Qu, D.; Xiao, L.; Tang, H.; Deng, Z.; Li, Y.; Su, B.-L. Self-assembly of polyhedral oligosilsesquioxane (POSS) into hierarchically ordered mesoporous carbons with uniform microporosity and nitrogen-doping for high performance supercapacitors. Nano Energy 2016, 22, 255-268. [CrossRef]

33. Tang, H.; Zeng, Y.; Gao, X.; Yao, B.; Liu, D.; Wu, J.; Qu, D.; Liu, K.; Xie, Z.; Zhang, H.; et al. Octa(aminophenyl)silsesquioxane derived nitrogen-doped well-defined nanoporous carbon materials: Synthesis and application for supercapacitors. Electrochimica Acta 2016, 194, 143-150. [CrossRef] 
34. Zhuang, X.; Zhang, Y.; He, L.; Zhu, Y.; Shi, Q.; Wang, Q.; Song, G.; Yan, X.; Li, L. Strategy to form homogeneously macroporous $\mathrm{Si}$ as enhanced anode material of Li-ion batteries. J. Alloys Compd. 2018, 731, 1-9. [CrossRef]

35. An, W.; Fu, J.; Su, J.; Wang, L.; Peng, X.; Wu, K.; Chen, Q.; Bi, Y.; Gao, B.; Zhang, X. Mesoporous hollow nanospheres consisting of carbon coated silica nanoparticles for robust lithium-ion battery anodes. J. Sources 2017, 345, 227-236. [CrossRef]

36. Guo, L.; He, H.; Ren, Y.; Wang, C.; Li, M. Core-shell SiO@F-doped C composites with interspaces and voids as anodes for high-performance lithium-ion batteries. Chem. Eng. J. 2018, 335, 32-40. [CrossRef]

37. Han, F.-D.; Bai, Y.-J.; Liu, R.; Yao, B.; Qi, Y.-X.; Lun, N.; Zhang, J.-X. Template-Free Synthesis of Interconnected Hollow Carbon Nanospheres for High-Performance Anode Material in Lithium-Ion Batteries. Adv. Mater. 2011, 1, 798-801. [CrossRef]

38. Yi, Y.; Lee, G.-H.; Kim, J.-C.; Shim, H.-W.; Kim, D.-W. Tailored silicon hollow spheres with Micrococcus for Li ion battery electrodes. Chem. Eng. J. 2017, 327, 297-306. [CrossRef]

39. Li, Z.; Li, Z.; Zhong, W.; Li, C.; Li, L.; Zhang, H. Facile synthesis of ultrasmall Si particles embedded in carbon framework using Si-carbon integration strategy with superior lithium ion storage performance. Chem. Eng. J. 2017, 319, 1-8. [CrossRef]

40. Wang, Z.-L.; Xu, D.; Wang, H.-G.; Wu, Z.; Zhang, X.-B. In Situ Fabrication of Porous Graphene Electrodes for High-Performance Energy Storage. ACS Nano 2013, 7, 2422-2430. [CrossRef]

41. Hu, C.; Wang, L.; Zhao, Y.; Ye, M.; Chen, Q.; Feng, Z.; Qu, L. Designing nitrogen-enriched echinus-like carbon capsules for highly efficient oxygen reduction reaction and lithium ion storage. Nanoscale 2014, 6, 8002. [CrossRef] [PubMed]

42. Bruce, P.; Saidi, M. The mechanism of electrointercalation. J. Electroanal. Chem. 1992, 322, 93-105. [CrossRef]

43. Kobayashi, S.; Uchimoto, Y. Lithium Ion Phase-Transfer Reaction at the Interface between the Lithium Manganese Oxide Electrode and the Nonaqueous Electrolyte. J. Phys. Chem. B 2005, 109, 13322-13326. [CrossRef] [PubMed]

44. Nakayama, M.; Ikuta, H.; Uchimoto, Y.; Wakihara, M. Study on the AC Impedance Spectroscopy for the Li Insertion Reaction of LixLa1/3NbO3at the Electrode-Electrolyte Interface. J. Phys. Chem. B 2003, 107, 10603-10607. [CrossRef]

45. Srivastav, S.; Xu, C.; Edstrom, K.; Gustafsson, T.; Brandell, D. Modelling the morphological background to capacity fade in Si-based lithium-ion batteries. Electrochimica Acta 2017, 258, 755-763. [CrossRef]

46. Li, X.; Zhu, X.; Zhu, Y.; Yuan, Z.; Si, L.; Qian, Y. Porous nitrogen-doped carbon vegetable-sponges with enhanced lithium storage performance. Carbon 2014, 69, 515-524. [CrossRef]

47. Qie, L.; Chen, W.-M.; Wang, Z.-H.; Shao, Q.-G.; Yuan, L.-X.; Hu, X.-L.; Zhang, W.-X.; Huang, Y.-H.; Chen, W.; Wang, Z; et al. Nitrogen-Doped Porous Carbon Nanofiber Webs as Anodes for Lithium Ion Batteries with a Superhigh Capacity and Rate Capability. Adv. Mater. 2012, 24, 2047-2050. [CrossRef] [PubMed]

48. Song, H.; Li, N.; Cui, H.; Wang, C. Enhanced storage capability and kinetic processes by pores- and hetero-atoms- riched carbon nanobubbles for lithium-ion and sodium-ion batteries anodes. Nano Energy 2014, 4, 81-87. [CrossRef]

49. Liu, Y.; Yan, X.; Yu, Y.; Yang, X. Self-improving anode for lithium-ion batteries: continuous interlamellar spacing expansion induced capacity increase in polydopamine-derived nitrogen-doped carbon tubes during cycling. J. Mater. Chem. A 2015, 3, 20880-20885. [CrossRef]

(C) 2019 by the authors. Licensee MDPI, Basel, Switzerland. This article is an open access article distributed under the terms and conditions of the Creative Commons Attribution (CC BY) license (http://creativecommons.org/licenses/by/4.0/). 